\title{
International Journal of Dentistry and Oral Health
}

\section{Reconstruction of Mandibular Alveolar Ridge Defects for Implant Placement: Critical Review}

\section{Ayman F. Hegab*}

Department of Oral and Maxillofacial Surgery, Faculty of Dental Medicine, Al-Azhar University in Cairo, Egypt

"Corresponding author: Ayman F. Hegab, Clinical Associate professor of Oral \& Maxillofacial Surgery, Department of Oral \& Maxillofacial Surgery, Faculty of Dental Medicine, Al-Azhar University in Cairo, Egypt, Tel: 00201001981112; E-mail: hegab@mail.com
Received date: 28 July 2015; Accepted date: 23 Sept 2015; Published date: 30 Sept 2015.

Citation: Hegab AF (2015) Reconstruction of Mandibular Alveolar Ridge Defects for Implant Placement: Critical Review. Int J Dent Oral Health 1(6): doi http://dx.doi.org/10.16966/2378-7090.138

Copyright: $\odot 2015$ Hegab AF. This is an open-access article distributed under the terms of the Creative Commons Attribution License, which permits unrestricted use, distribution, and reproduction in any medium, provided the original author and source are credited.

\begin{abstract}
Background: Dental rehabilitation of partially or totally edentulous patients with oral implants has become a routine treatment modality in the last decades, with reliable long-term results. However, unfavorable local conditions of the alveolar ridge, due to atrophy, periodontal disease, and trauma sequelae may provide insufficient bone volume or unfavorable vertical, horizontal, and sagittal intermaxillary relationships, which may render implant placement impossible or incorrect from a functional and esthetic viewpoint. The aim of the current review is to discuss the different strategies for reconstruction of the alveolar ridge defect for implant placement.
\end{abstract}

Study design: The study design includes a literature review of the articles that address the association between Reconstruction of Mandibular Alveolar Ridge Defects and Implant Placement.

Results: Yet, despite an increasing number of publications related to the correction of deficient alveolar ridges, much controversy still exists concerning which is the more suitable and reliable technique. This is often because the publications are of insufficient methodological quality (inadequate sample size, lack of well-defined exclusion and inclusion criteria, insufficient follow-up, lack of well-defined success criteria, etc.).

Conclusion: On the basis of available data it is difficult to conclude that a particular surgical procedure offered better outcome as compared to another. Hence the practical use of the available bone augmentation procedures for dental implants depends on the clinician's preference in general and the clinical findings in the patient in particular. Surgical techniques that reduce trauma, preserve and augment the alveolar ridge represent key areas in the goal to optimize implant results.

Keywords: Mandibular reconstruction; Dental implant; Bone graft; Distraction osteogenesis; Bone Manipulation; Bone bioengineering.

\section{Introduction}

Reliable rehabilitation of the alveolar ridge with endosseous implants requires proper quality and quantity of alveolar bone at the implant site in order to achieve a good long term prognosis. Sufficient bone volume is still considered the most important prerequisite for predictable long term prognosis for dental implant [1].The placement of implants requires sufficient volume of sound bone in which to place the fixtures [2].

The failure rate of short implants is higher than that of longer implants and complications are more common. Insufficient bone height often means that the crown height: implant length ratio is too great, which is likely to reduce the implant's useful life span, because of the leverage effect (degree of force acting on the implant site). It has been suggested that the length of the crown should not be more than $50 \%$ of total length of the prosthesis [3].

The minimum implant length (i.e. bone height) in an ideal bone density situation for predictable success is $10 \mathrm{~mm}$. allowing a margin of 2 $\mathrm{mm}$ from the vital landmarks like inferior alveolar canal is recommended. Available width is defined as the distance between the buccal and lingual plates, measured at the crest. Each $1 \mathrm{~mm}$ increase in diameter increases the surface area by about 20 to $30 \%$ therefore increasing diameter effectively decreases crestal stress. Thus implant diameter is much more critical than its length. Implant length, on the other hand, only improves initial stability [1].

A multidimensional assessment of the available bone is the most important factor for a sound treatment planning ensuring longevity and functionality of the dental implants. Bone measurements need to be made in 3 axes: the $\mathrm{X}$ axis: mesio-distally; $\mathrm{Y}$ axis: vertically; and $\mathrm{Z}$ axis: buccolingually. Unfortunately the vast majority of the patients who request restorations for missing teeth do exhibit some degree of bony deficiency by the time they present for their treatment. Alveolar ridge deficiencies may be seen in the vertical or horizontal axes, or commonly in both.

When the bone is compromised in the $\mathrm{X}$ axis, it can be corrected by orthodontic tooth movement. While, when the bone is compromised in the $\mathrm{Y}$ and $\mathrm{Z}$ axis and to enable placement of an implant, augmentation becomes necessary to increase the bone volume.

Teeth extraction is the main cause of alveolar ridge atrophy. Teeth may have been lost through dental disease or trauma or they may be congenitally absent. In addition, teeth may be lost as part of a surgical procedure to resect part of a jaw because of pathology such as cancer. Non-extraction aetiologies of alveolar bone loss includes denture-induced atrophy, trauma, periodontal disease, congenital alveolar defects, and tumour resection [4].

Different classifications to describe alveolar ridge defects have been published in the dental literature. The various extant defect classifications address only a subset of the possible hard-tissue defect situations, largely disregarding the overall intraoral situation and the environment of the defect. In addition, most of the current classifications didn't address the relation between the deficiency and the reconstruction.

In 1963, Atwood [5] described 6 classes of alveolar ridge atrophy: Atwood included in his anatomic classification the ridge resorption in a 
horizontal direction. Measurements were made in a cross section of the mandible at the site of the central incisor.

I. Pre-extraction normal bone.

II. Post-extraction normal bone: after extraction and before resorption started.

III. High well rounded, adequate in height and width.

IV. Knife-edge, adequate height, inadequate width.

V. Low, well rounded, inadequate height and width.

VI. Depressed ridge

Yet, it would appear obvious that, for example, the number of walls delimiting the defect and their relationship to the overall jaw situation significantly impacts the extent of treatment required as well as the postaugmentation success rates. Small, localized defects with ideally shaped hard tissue possibly bordering on still existing adjacent teeth or ridge areas and located within the jawbone geometry ("within the contour") are therefore easier to reconstruct and stabilize. Furthermore, localized defects have advantages in terms of higher regenerative capacity (originating from the defect floor), smaller volume and lower soft-tissue pressure [6].

\section{Review of Literature}

\section{Treatment options for alveolar ridge defects}

During the past decade, ridge augmentation techniques have gained much attention in pre-implant surgery. Various augmentation techniques are currently in use to create sufficient bone volume for reliable placement of endosseous implants in the case of severely resorbed alveolar ridges.

As the field of the implant dentistry is dynamic, many clinicians are searching for pre-implant surgical procedures that are less inconvenient to the patients and still possess the ability to create optimal circumstances for implant placement.

Treatment of alveolar ridge defect consisted of four strategies; Bone replacement grafts, bone manipulation procedures, distraction osteogenesis and Bone bioengineering.

Selection of the surgical procedures for reconstruction of the alveolar ridge defect depends on many factors; site and size of the defect, intermaxillary relationships, donor site morbidity, thickness and height of the available bone, biological properties of the respective bone substitute material, and soft tissue deficiencies [7].

\section{Bone replacement grafts}

Bone replacement grafts (bone grafts and bone graft substitutes) provide a structural framework for clot development, maturation and remodelling that supports bone formation in osseous defects.Several categories of bone graft and graft substitutes exist and encompass a variety of materials, material sources, and origins. The available graft substitutes formed from composites of one or more types of material. These composites are generally built on a base material. Laurencin et al. [8] classification of grafts and graft substitutes as follows:

Harvested bone grafts and graft substitutes: Bone grafts, endogenous or exogenous, are often essential to provide support, fill voids, and enhance biologic repair of skeletal defects. Limitations of use of endogenous bone substance involve additional surgery; often resulting donor site morbidity and limited availability. Whereas, allograft have been encountered with risk of disease transmission, immunogenicity. Therefore, there is a growing need for synthesis of allograft bone substitutes used alone or in combination with other materials (e.g., Allogro (AlloSource, Centennial, Colo), Opteform (Exactech, Inc, Gainesville, Fla), Grafton (BioHorizons, Birmingham, Ala), OrthoBlast (IsoTisOrthoBiologics, Irvine, Calif)) [9].
Growth factor-based bone graft substitutes: Natural and recombinant growth factors used alone or in combination with other materials such as transforming growth factor-beta (TGF-beta), platelet-derived growth factor (PDGF), fibroblast growth factor (FGF), and bone morphogenetic protein (BMP).

Cell-based bone graft substitutes: Use cells to generate new tissue alone or are seeded onto a support matrix (e.g., mesenchymal stem cells).

Ceramic-based bone graft substitutes: Include calcium phosphate, calcium sulfate, and bioglass used alone or in combination (e.g., OsteoGraf (DENTSPLY FriadentCeraMed, Lakewood, Colo), Norian SRS (Synthes, Inc, West Chester, $\mathrm{Pa}$ ), ProOsteon [Interpore Cross International, Irvine, Calif), Osteoset (Wright Medical Technology, Inc, Arlington, Tenn)).

Polymer-based bone graft substitutes: Degradable and nondegradable polymers are used alone or in combination with other materials (e.g., Cortoss (Orthovita, Inc, Malvern, $\mathrm{Pa}$ ), open porosity polylactic acid polymer (OPLA), Immix (Osteobiologics, Inc, San Antonio, Tex)).

Miscellaneous: Various unconventional marine biomaterials are also in use as bone graft substitute which includes coral, chitosan, sponge skeleton etc.

Bone grafts and their substitutes can also be divided into osteoinductive agents, osteoconductive agents and osteogenic agents.

Osteoinductive agents are generally proteins, which induce differentiation of undifferentiated stem cells to osteogenic cells or induce stem cells to proliferate

Osteoconductionis the process whereby microscopic and macroscopic scaffolding is provided for inward migration of cellular elements involved in bone formation (e.g., mesenchymal cells, osteoblasts, osteoclasts, and vasculature).

Osteogenesis in a general sense, osteogenesis refers to bone formation with no indication of cellular origin: new bone may originate from live cells in the graft or cells of host origin [10].

Autogenousbone grafts are still considered the gold standard inbone regeneration procedures. However, donor sitemorbidity, unpredictable resorption, limited quantitiesavailable, and the need to include additional surgicalsites are drawbacks related to autografts thathave intensified the search for suitable alternatives. Bone-substitute materials have increased in popularityas adjuncts to or replacements for autografts inbone augmentation procedures to overcome the limitationsrelated to the use of autografts [7].

Autogenous bone harvesting is the acquisition of a specific quantity, quality, and contour of bone required for the reconstruction. Many donor sites are available for obtaining small to moderate volumes of bone for maxillofacial reconstruction. These include the calvarium, iliac crest, the rib, tibia, mandibular symphysis, coronoid process, maxillary tuberosity and so on [11-19].

These harvest sites differ on the basis of their embryological characteristics (endochondral vs. intramembranous ossification), types of bone (cancellous vs. cortical), morphological and physical characteristics, the morbidity associated with harvest from the specific donor site, the volume of graft to be obtained, and the rate of their resorption. The desirable characteristics of a bone graft are sufficient volume, minimal donor-site morbidity, obtaining intramembranous bone with high cortical component, proximity to the recipient site, ease of harvesting and achieving of reproducibleand good results, and minimal resorption rate [20].

The choice of autogenous donor site is markedly influenced by two important considerations; namely, the quantity of bone required at the 
recipient site and the biologic qualities of the donor bone. Additionally, successful augmentation of the recipient site is influenced by the technical, intraoperative surgical manipulations employed. It is readily apparent that the quantity of bone required is a major factor in donor site selection. An extraoral donor site is often required for ridge augmentation in totally edentulous patients [7].

Intraoral sites generally allow for shorter procedures, avoid the need for general anesthesia, and are associated with few complications and less postoperative discomfort. Somewhat less apparent than the bone quantity required, but no less important, are the biologic qualitiesof the transplanted bone. These include the bone's embryologic origin, morphology, cytological constituents, and biochemical composition of the extracellular matrix [21]

The mandibular symphysis is almost invariably, however, not contiguous with the area to be augmented. This requires the involvement of a second surgical site. Clearly, an alternative mandibular donor site that is contiguous with the recipient area would obviate the need for an extra surgical site. Such alternative sources for local harvesting in the mandible can be evaluated by careful clinical and radiographic examinations of the patient; the mandibular ramus has some advantages when compared to the mental symphysis as a possible donor site: the quality of bone is similar, the quantity may be higher, and the risk of neural damage is lower [21].

For larger defects, distant or extraoral sites are preferably employed. The iliac crest is the most common donor site because of easy access and procurement, low morbidity, and availability of large quantities of both cortical and cancellous bone. Harvesting of iliac crest bone graft is associated with some potentially serious complications. The most common symptoms attributable to the donor site are pain and sensory disturbances. Variations in patient population, diagnosis, procedure, surgical technique, and study design contribute to a broad range of reported complications related to iliac crest donor sites [7].

During the last two decades the use of the cranial bone grafts for maxillofacial procedures has been accepted. Its successful use at various craniomaxillofacial sites. Both cortical and cancellous bone can be harvested from the calvarium in young patients, and the different instruments and techniques that are available allow various consistencies to be harvested [7].

The limitations associated with the procurement of autograft for bone grafting can be overcome by the use of allografts. Allograft bone is referred to as cadaver, obtained from donor bone and has both osteoinductive (they release bone morphogenic proteins that act on bone cells) and osteoconductive properties, but lack osteogenic properties because of the absence of viable cells. However, harvesting and conservation of allogenic grafts are additional limiting factor [10].

The major advantage of allograft bone harvested from cadaver sources include its ready availability in various shapes and sizes, avoidance of the need to sacrifice host structures, and no donor site morbidity. Still, there is some controversy regarding association of allograft bone with the transmission of infectious agents, a major concern virtually eliminated through tissue-processing and sterilization [10,22]. Allogenic bone is available in many forms: demineralized bone matrix, cancellous chips, corticocancellous and cortical grafts, and osteochondral and whole-bone segments [10]. With introduction ofGuided bone regenerationtechnique, combination of block grafts with bone fillers and membranes was applied. The first membrane used for horizontal ridge augmentation was expanded polytetrafuroethylene (ePTFE), but because of the increased risk of complications following wound dehiscence, resorbable collagen membrane introduced $[23,24]$.
The concept of interpositional or "sandwich" grafting is based on the theory that bone placed between 2 pieces of pedicled bone with internal cancellous bone will undergo rapid and complete healing and graft incorporation. Schettler [25] proposed the "sandwich-technique" for vertical augmentation of the mandible, involving a horizontal osteotomy of the mandible, leaving the lingual soft tissue attachments. The cephalic bone was raised, and autogenous grafting material was inserted in the defect, healing with minimal bone resorption, regardless of the interpositional graft material used.

Interpositional osteotomies in the alveolar bone heal with rapid vascularization and bone remodelling in the bone gap. After 12 weeks, the interpositional grafts were almost indistinguishable from the surrounding native bone. When performing an interpositional osteotomy and moving the mobilized alveolar bone segment vertically, the clinician must decide on the optimal material to graft the defect. Cancellous/particulate marrow grafts have shown more rapid vascularization and more osteogenic activity compared with autogenous block grafts [26].

\section{Bone Manipulation Procedures}

Bone expansion can be defined as the manipulation of the bone to form a receptor site for an implant without the removal of any bone from the patient. Many people incorrectly believe that bone is hard and unbendable. However Living bone is much softer and can be stretched open, making the concept of bone expansion possible. Through the serial use of graduated chisel-like cylindrical or tapered instruments ridges can be slowly expanded to increase their width [1]

\section{Rationale behind Bone Expansion Techniques}

Ridge splitting and expansion makes use of visco-elastic properties of bone. Bone is a biologically privileged tissue in that it has the capacity to undergo regeneration as a part of the repair process. When the clinician is persevering and allows ample time for the manipulation of the bone, it can eventually be modeled towards the desired location. Careful manual manipulation is more important than merely tapping with the surgical mallet [27].

Displacement of the osseous segment results in positioning of a healthy portion of bone into a previously deficient site. Because the soft tissue is left attached to the transported segment, the movement of the bone also results in expansion of the soft tissue adjacent to the bone segment, improving both the hard and soft tissue contour. At the original location of the segment is left a regeneration chamber which has a natural capacity to heal by filling with bone, instead of fibrous tissue. This is a function of the surrounding, healthy cancellous bone walls and location within the skeletal functional matrix. As a result, the alveolar housing, including the osseous and soft tissue components is enlarged in a single, simultaneous process [28].

This technique will permit placement of regular sized implants through the expanded ridge crest. This bone segment is not regenerated or grafted tissue; it is native, mature bone which is an ideal situation to deal with. The success of the bone manipulation procedures relies on maintaining the integrity of the labial wall, which occurs as long as the periosteum remains intact. Since $80 \%$ of the blood supply is from the periosteum, we feel the high degree of the success in expanding very thin ridges is due to our ability to manipulate the thin cortical bone without disrupting the periosteal attachment to this bone $(27,28)$

So often during bone splitting or expansion, the periosteum is not raised and it remains encasing the bone to provide blood supply and physical support. Its elastic nature allows the bone to spread and expand and at the same time contains all the micro-fractures that may occur. In fact intact periosteum acts as a barrier membrane and makes the fractures heal very well because of the intact blood supply [29]. 


\section{Objective of Ridge Expansion}

The lateral ridge expansion technique is aimed at the creation of a new implant bed by longitudinal osteotomy of the alveolar bone. The buccal cortex is repositioned laterally using a greenstick fracture, and the space between the buccal and lingual cortical plates is filled with autologous, allogenic, alloplastic graft material or without any graft material. The lateral ridge expansion technique is usually performed simultaneously with implant placement and significantly shortens the treatment time [27].

In the ridge splitting procedure, the corticotomies can be performed using sharp chisel, round bur, fissure bur, diamond disk, reciprocal saw, or piezoelectric device or laser (erbium: yttrium-aluminum-garnet, erbium, chromium-doped: yttrium-scandium-gallium-garnet) [27].

The piezoelectric saw is safer and more precise than the conventional rotary bur or reciprocal saw when performing osteotomies. Piezoelectric devices use ultrasonic microvibrations to create an osteotomy, and these microvibrations make selective bone cuts possible without soft tissue damage. Moreover, more precise cuts can be performed and a severely narrow ridge of 2 to $3 \mathrm{~mm}$ can be osteotomized [29].

The erbium: yttrium-aluminum-garnet laser allows increased haemostasis, improved visibility during surgery, a reduced incidence of infection, and reduced patient discomfort postoperatively when applied to soft and hard tissue [30].

In the mandible, the risk of malfracture of the osteomized segment is great because the mandibular bone has less flexibility and a thicker cortical plate. Ridge expansion with simultaneous implant placement has resulted in several complications such as a lack of initial stability for the implants, fracture of the buccal segmented bone, and compromised implant placement in the buccolingual and apicocoronal direction [29].

The lateral ridge expansion technique is very effective for horizontal augmentations in severely atrophic posterior mandibular ridges. In the mandibular ridge, which has low bone quality and a thin cortex, immediate lateral ridge expansion can be a useful procedure. Delayed lateral ridge expansion can be used more safely and predictably in patients with high bone quality and a thick cortex and narrower ridge in the mandible to avoid complete fracture of the buccal segments. In addition, delayed ridge expansion is recommended when the initial stability of the implants is poor [27].

\section{Distraction Osteogenesis}

In alveolar DO, in line with the basic principles of tissue engineering, gradual traction of the pedicled bone fragment is followed by simultaneous osteogenesis (bone) and histogenesis (functional soft tissue matrix). This permits shortening of the overall treatment period and earlier placement of implants which show success rates comparable to implants placed in native alveolar bone [31].

In view of its numerous advantages, DO may be considered a treatment option in diverse conditions unfavorable for implant placement, but both basic and clinical research demonstrates that alveolar DO still has significant limitations. After many years of widespread application of DO in the alveolar ridge there is still significant disagreement about various treatment parameters, such as surgical technique, type of distraction device and minimal bone height necessary to perform the distraction. There is a lack of evidence regarding appropriate DO protocols, maximum possible augmentation distance and long-term implant success rates. In the posterior region, DO was applied more frequently in the mandible than in the maxilla, to permit the placement of implants with increased length and reduced crown height [31].
The surgical procedure for vertical alveolar bone distraction is relatively simple. However, some problems have been encountered during distraction due to surgical technique or anatomical situations. The first problem is resistance during rotation of the device. This phenomenon is partly due to tension from the soft tissue but mainly due to lateral friction of the bone surface between the transport segment and the alveolar bone, especially when the vertical osteotomy line is formed in a parallel shape rather than a divergent shape toward the alveolar crest [31].

In some commercial intraosseous distractors, the transport segment is unstable and this floating segment may be displaced to the lingual side during the distraction period. This is because the resistance of the soft tissue on the lingual side of the muscles on the floor of the mouth, and intact periosteum [31].

The surgical procedure for horizontal alveolar bone distraction (widening) is more complicated than the vertical distraction. Alveolar widening by DO has not become a standard procedure. The main reason for this may be the small number of commercially available distractors and the small number of clinical reports [32].

In fact, only a few alveolar widening distractors are commercially available. Most reported techniques for alveolar widening are designed based on the same concept. With this concept, the anchorage for the distraction screw, which penetrates the distraction segment, is made at the palatal or lingual cortex and the segment moves toward the lateral side by twisting off movements of the screw-rod. Watzak et al. showed a simple procedure based on the above concept in which the penetrated long mini screw simply moves the segment by the cortex anchorage [32].

\section{Future Perspective}

In contrast to the favourable aspects of autologous bone, the search for alternatives has been motivated by the drawbacks of the harvesting procedure. A challenging, biological driven method is to mimic a bone to allow the development of a three-dimensional autologous bone substitute.

Tissue Engineering blends regenerative medicine and surgery, with its three basic components being scaffold, cells, and signaling molecules. Tissue regeneration and functional restoration are achieved through the implantation of cells and tissue developed outside the body or the promotion of cells growth in an implanted matrix.

Stem cells play vital roles in the repair of every organ and tissue through their capacity for self-renewal and differentiation. Stem cells can self-renew and produce different cell types, thus providing new strategies to regenerate missing tissues and treat diseases [33-38].

Mesenchymal stem/stromal cells (MSCs) are now an excellent candidate for tissue replacement therapies, and tissue engineering approaches and chair-side cellular grafting approaches using autologous MSCs represent the clinical state of the art for stem-cell-based alveolar bone regeneration. Basic studies have revealed that cross talk between implanted donor cells and recipient immune cells play a key role in determining clinical success that may involve the recently observed immunomodulatory properties of MSCs [33-38].

In the field of dentistry, especially in the clinic, material-based reconstruction without major surgical procedures was the main approach to treatment; however, emerging stem cell technologies and the requirements of alveolar ridge augmentation associated with implant dentistry have expanded the clinical concept to include stem-cell-based regeneration. Stem cell technologies have even permitted dental scientists to imagine the development of bioengineered teeth to replace the patient's missing teeth. Furthermore, "dental stem cell banking" is already on the market for possible future use in regenerative therapies [33-38]. 


\section{Management of Horizontal Ridge Defects}

These cases have adequate alveolar bone height but inadequate width. The deficiency is most commonly on the facial surface of the mandible. The planning of these cases should include physical and radiographic examination. The site and the planned implant diameter dictate the amount of bone required. For most segmental deficiencies in the mandible, autogenous grafts can be harvested from theSymphysis or ramus $[39,40]$.

Recipient site preparation is critical for predictable incorporation of block graft and includes decortication and perforation into underlying marrow. This preparation provides access for trabecular bone blood vessels to the graft and accelerates revascularization. There is also massive platelet release along with associated growth factors and osteogenic cells. Finally, graft union to the underlying host bone is accomplished more readily, which allows for intimate contact to facilitate graft incorporation [11].

Decortication creates an outline for close graft approximation. Bone burnishing with a large round bur from crest of ridge to approximately 4 to $5 \mathrm{~mm}$ apically is done initially. Decortication continues apically with a straight fissure bur in a more aggressive fashion to create extra walls to the defect in the form of a rectangular inlay preparation [11].

With localized horizontal augmentation, where the vertical height is adequate, and only width is lacking, particulate graft material can be used and protected with a membrane. To reconstruct horizontal deficiency of the mandibular alveolus with particulate graft, the principles of graft protection during the healing phase are important.The particulate graft is placed and then covered with a membrane. The choice of particulate graft material becomes important if a non-rigid membrane is used. The particulate graft must have some strength to resist deformation [39-41].

Established treatment modalities for horizontal defects include, in addition to augmentation with autologous bone, the use of boneexpansion (bone-splitting) techniques and guided bone regeneration (GBR). Bone expansion as sole treatment requires sufficiently flexible oral and vestibular bone lamellae, so this approach is suitable only where reconstructive needs are moderate.

For localized horizontal defects, GBR has shown results comparable to those obtained with autologous bone. Slow-resorbing bone substitutes and membranes or non-absorbable barrier membranes are recommended for more extensive GBR procedures and for augmentation outside the envelope of the ridge [24,42-44].

Note that higher infection rates were found when augmenting extended defects with non-absorbable membranes and bone substitutes than for autologous bone block grafts. Collagen membranes are associated with lower complication rates than non-absorbable membranes, but should maintain longer absorption time in the case of larger augmentation volumes. To improve the osteogenic potential of the augmentation material, the admixture of natural bone (e.g. chips obtained while preparing the implant bed) is recommended. For medium and extended augmentation outside the contour, "tent-pole technique", may be considered [23,24,42-45].

The edentulous horizontally deficient mandible can be treated with multiple local grafts or bone from distant sites. Because of the large amount of bone needed, harvest from a distant site is usually indicated.

Distraction osteogenesis can be used via different techniques.Ridge widening using punch-tip pilots or implant analogue can be used successfully. This procedure expands the alveolar ridge by using successive wedging of smooth surface implant analogue that is inserted in between the 2 surgically split cortices and driven in by circular motion.

Transverse alveolar distraction devices include the following; Laster crest device, Multidirectional osteodistraction device, and Extension crest device.

\section{Laster crest device}

This device consists of 2 parallel metal arches; each has 1 horizontal plate and 2 vertical sharp blades that are inserted into the alveolar crest. The 2 horizontal plates are connected together by 2 sliding pins and 2 laterally positioned activation screws. Activation gradually separates the 2 arches, thereby expanding the alveolar crest [46].

\section{Multidirectional osteodistraction device}

This tooth-supported device can provide simultaneous vertical and horizontal distraction if needed. Bicortical bone segment is separated from the alveolar ridge by 1 horizontal and 2 vertical osteotomies. Then, intraosseous anchor abutments are inserted into the bone segment, anchored against the basal bone. Activation of the device results in controlled vertical and horizontal movement of the bone segment relative to the surrounding teeth onto which the device is anchored [47].

\section{Extension crest device}

This device consists of 2 arms that are apically hinged together and crestally connected by a threaded pin that passes transversely through 1 arm to abut against the other. Activation of the screw pushes the crestal ends of 1 arm away from the other around the apical hinge. The device is installed vertically through the alveolar crest after splitting the buccal cortex from the lingual by 2 vertical osteotomies connected by a horizontal crestal osteotomy [47].

\section{Management of Vertical Ridge Defects}

Vertical augmentation of the alveolar ridge is more difficult than horizontal augmentation. There is concern when expanding the soft tissue envelope vertically there is an increased risk of graft exposure. A second concern is the adequate adaptation of the bone graft, which is critical for graft success [11].

Site preparation for vertical augmentation requires only crestal bone burnishing to create bone bleeders followed by perforations into marrow. Many of these alveolar ridges are deficient in height and width and may require flattening for better graft adaptation. If thin cortical grafts are harvested, then a stack technique is used to achieve the desired result. This technique involves the mortising of the graft to the ridge surface and the placement of multiple block grafts stacked on one another to achieve height. The graft is secured with a bone screw or by dental implants. A second technique is to augment the defect with a single cortico-cancellus block that adequately replaces the desired amount of bone [11].

Due to the increased difficulty of soft-tissue management and the need to stabilize the augmentation material, treating vertical defects is more demanding than treating strictly horizontal defects. Possible options include, in addition to autologous bone block graft, stabilizing systems such as titanium-reinforced ePTFE membranes, - positioning screws (tent-pole technique) or screw fixations for allogenous blocks [23,44-45].

Osteoconductive bone substitutes in onlay apposition technique may be used in combination with autologous bone and non-absorbable membranes, and remain limited to situations with minor vertical augmentation requirements. Larger defects may be treated with application of biomaterials in sandwich-technique, where bone formation is supported from both crestal and basal bone matrix after horizontal split osteotomy [42,44-45].

Interpositional osteotomies in the alveolar bone heal with rapid vascularization and bone remodelling in the bone gap. After 12 weeks, the interpositional grafts were almost indistinguishable from the surrounding native bone [26].

When performing an interpositional osteotomy and moving the 
mobilized alveolar bone segment vertically, the clinician must decide on the optimal material to graft the defect. Cancellous/particulate marrow grafts have shown more rapid vascularization and more osteogenic activity compared with autogenous block grafts [26]

The surgical procedure for vertical alveolar bone distraction is relatively simple. However, some problems have been encountered during distraction due to surgical technique or anatomical situations. The first problem is resistance during rotation of the device. This phenomenon is partly due to tension from the soft tissue but mainly due to lateral friction of the bone surface between the transport segment and the alveolar bone, especially when the vertical osteotomy line is formed in a parallel shape rather than a divergent shape toward the alveolar crest [49].

In some commercial intraosseous distractors the transport segment is unstable and this floating segment may be displaced to the lingual side during the distraction period. This is because the resistance of the soft tissue on the lingual side of the muscles on the floor of the mouth, and intact periosteum [49].

Distraction osteogenesis for the molar region using existing commercial devices has limited indications, such as difficult control of direction, occlusal disturbance from the device, instability of transport segment, loss of callus at second surgery, and infection through the device, which may lead to unsatisfied results. Moreover, some of these distractors cannot be used at the molar region of severe mandibular atrophy because minimum height of bone above mandibular canal is needed for placement of the device [49].

\section{Management of Combined Defects}

Bone defects that arise after dental extraction usually result in both horizontal and vertical bone loss. With time, the soft tissue shrinks concomitantly with the bone, making large augmentations difficult.

Bone grafting is one of the methods for augmentation of the combined (vertical and horizontal) defects. Nevertheless, there are two main limitations of bone grafting; one is the lack of soft tissue to cover the new volume of bone resulting in graft exposure and the second is lack of surface area in contact with the graft to allow incorporation and revascularization. This means increase the graft volume result in greater distance of the surface of the graft from a blood supply.

The autogenous J-graft addresses both dimensions (vertical and horizontal) in the localized combined alveolar ridge defects. While in case of extensive combined ridge defects, more complicated graft procedures will require [50].

The problem of lack of enough soft tissue to cover the graft can be overcome by the distraction osteogenesis. When performing distraction osteogenesis of combined defects, some overexpansion with subsequent subtractive adjustment of the compromised horizontal areas is feasible. Extensive horizontal and vertical bone resorption without bone walls can usually only be treated with autologous bone grafts, since the augmentation bed will not offer enough osteogenic potential for substitute material application only.

\section{Conclusions}

On the basis of available data it is difficult to conclude that a particular surgical procedure offered better outcome as compared to another. Hence the practical use of the available bone augmentation procedures for dental implants depends on the clinician's preference in general and the clinical findings in the patient in particular.

Surgical techniques that reduce trauma, preserve and augment the alveolar ridge represent key areas in the goal to optimize implant results.
Future biosynthetic bone implants may obviate the need for autologous bone grafts. There is increasing interest in combining an osteoconductive protein in an osteoconductive carrier medium to facilitate timed-release delivery and/or to provide a material scaffold for bone formation. Further, advances in tissue engineering, "the integration of the biological, physical and engineering sciences" will generate new carrier constructs that repair, regenerate and restore tissue to its functional state.

Finally, as researchers continue to find new materials and biologic approaches to bone repair, the future of bone graft substitutes continues to be an expanding topic of interest.

\section{References}

1. Goyal S, lyer S (2009) Bone Manipulation Techniques. Int J Clini Implant Dentistry 1: 22-31.

2. Misch k, Wang H (2008) Implant Surgery Complications: Etiology and Treatment. Implant Dentistry 17: 158-169.

3. Sakka S, Baroudi K, Nassani M (2012) Factors associated with early and late failure of dental implants. J Investig Clin Dent 3: 258-61.

4. Esposito M, Grusovin MG, Felice P, Karatzopoulos G, Worthington HV, et al. (2009) Interventions for replacing missing teeth: horizontal and vertical bone augmentation techniques for dental implant treatment (Review).Cochrane Database Syst Rev 7: CD003607.

5. Atwood DA (1963) Postextraction changes in the adult mandible as illustrated by microradiographs ofmidsagittal sections and cephalometric roentgenograms. J Prosthetic Dent 13: 810-24.

6. Ehrl P, Fürst U, Happe A, Khoury F, Kobler P, et al. (2013) Cologne Classification of Alveolar Ridge Defects (CCARD). Consensus paper approved at the 8th European Consensus Conference of BDIZ EDI (EuCC) in Cologne, February 2013.

7. Chiapasco M, Casentini P, Zaniboni M (2009) Bone augmentation procedures in implant dentistry. Int J Oral Maxillofac Implants. 24: 237-59.

8. Laurencin C, Khan Y, El-Amin SF (2006) Bone graft substitutes. Expert Rev Med Devices 3: 49-57.

9. Pollock R, Alcelik I, Bhatia C, Chuter G, Lingutla K, et al. (2008) Donor site morbidity following iliac crest bone harvesting for cervical fusion: a comparison between minimally invasive and open techniques. Eur Spine J 17: 845-52.

10. Nandi S, Roy S, Mukherjee P, Kundu B, Basu D (2010) Orthopaedic applications of bone graft \& graft substitutes: a review. Indian J Med Res 132: 15-30.

11. Toscano N, Shumaker N, Holtzclaw D (2010) The Art of Block Grafting: A Review of the Surgical Protocol for Reconstruction of Alveolar Ridge Deficiency. The Journal of Implant \& Advanced Clinical Dentistry.

12. Yates D, Brockhoff II H, Finn R, Phillips C (2013) Comparison of Intraoral Harvest Sites for Corticocancellous Bone Grafts. J Oral Maxillofac Surg 71: 497-504.

13. Amrani S, Anastassov G, Montazem A (2010) Mandibular Ramus/ Coronoid Process Grafts in Maxillofacial Reconstructive Surgery. J Oral Maxillofac Surg 68: 641-646.I

14. De Riu G, Meloni M, Pisano M, Baj A, Tullio A (2012) Mandibular coronoid process grafting for alveolar ridge defects. Oral Surg Oral Med Oral Pathol Oral Radiol 114: 430-436.

15. Fasolis M, Boffano P, Ramieri G (2012) Morbidity associated with anterior iliac crest bone graft. Oral Surg Oral Med Oral Pathol Oral Radiol 114: 586-591.

16. Cuesta Gil M, Bucci T, Ruiz B (2010) Reconstruction of the severely atrophic mandible using autologous calvarial bone graft: An "inverted sandwich" graft technique and dental rehabilitation with fixed implantsupported prostheses. J Craniomaxillofac Surg 38: 379e384. 
17. Abdel-Haleem A, Nouby R, Taghian M (2011) The use of the rib grafts in head and neck reconstruction . Egyptian Journal of Ear, Nose, Throat and Allied Sciences 12: 89-98.

18. Walker T, Modayil P, Cascarini L, Williams L, Duncan S, et al. Retrospective review of donor site complications after harvest of cancellous bone from the anteriomedial tibia. $\mathrm{Br} \mathrm{J}$ Oral Maxillofac Surg 47: 20-22.

19. Tolstunov $L$ (2009) Maxillary tuberosity block bone graft: innovative technique and case report. J Oral Maxillofac Surg 67: 1723-1729.

20. Rawashdeh M, Telfah H (2008) Secondary Alveolar Bone Grafting: the Dilemma of Donor Site Selection and Morbidity. Br J Oral Maxillofac Surg 46: 665-670.

21. Brugnami F, Caiazzo A, Leone C (2009) Local Intraoral Autologous Bone Harvesting for Dental Implant Treatment: Alternative Sources and Criteria of Choice. Keio J Med 58: 24-28.

22. Marx RE (2007) Bone and bone graft healing. 28. Oral Maxillofac Surg Clin North Am 19: 455-66.

23. Kuo S, Niu G, Lan C, Cheng M, Chiang M, Chang S (2009) Guided Tissue Regeneration with Use of CaSO4-Chitosan Composite Membrane. J Med Biol Eng 29: 304-310.

24. Mihatovic I, Becker J, Golubovic V, Hegewald A, Schwarz F (2012) Influence of two barrier membranes on staged guided bone regeneration and osseointegration of titanium implants in dogs. Part 2: augmentation using bone graft substitutes. Clin Oral Impl Res 23: 308-315.

25. Schettler D (1976) Sandwich-technique with cartilage transplant for raising the alveolar process in the lower jaw. Fortschr Kiefer GesichtsChir 20:61.

26. Block M, Haggerty C (2009) Interpositional Osteotomy for Posterior Mandible Ridge Augmentation. J Oral Maxillofac Surg 67: 31-39.

27. Sohn D, Lee H, Heo J, Moon J, Park I, Romanos G (2010) Immediate and Delayed Lateral Ridge Expansion Technique in the Atrophic Posterior Mandibular Ridge. J Oral Maxillofac Surg 68: 2283-2290.

28. Elian N, Jalbout Z, Ehrlich B, Classi A, Cho S, et al. (2008) A twostage full-arch ridge expansion technique: Review of the literature and clinical guidelines. Implant Dent 17:16.

29. Sohn DS (2008) Color Atlas, Clinical Applications of Piezoelectric Bone Surgery. Kunja Publishing Group, Seoul, 2008.

30. Sohn DS, Lee JS, An KM, Romanos GE (2009) Erbium, chromium: yttriumscandium- gallium-garnet laser-assisted sinus graft procedure. Lasers Med Sci 24: 673.

31. Hegab AF, Shuman MA (2012) Distraction Osteogenesis of the Maxillofacial Skeleton: Biomechanics and Clinical Implications. Open Access Scientific Reports. 11: 1-10.

32. Watzak G, Zechner W, Tepper G, Vasak C, Busenlechner D, Bernhart T (2006) Clinical study of horizontal alveolar distraction with modified micro bone screws and subsequent implant placement. Clin Oral Implants Res 17: 723-729.

33. Koyano K (2012) Toward a new era in prosthodontic medicine. J Prosthodont Res 56: 1-2.
34. Egusa H, Saeki M, Doi M, Fukuyasu S, Matsumoto T, Kamisaki Y, et al. (2010) A small-molecule approach to bone regenerative medicine in dentistry. J Oral Biosci 52: 107-118.

35. Egusa H. iPS cells in dentistry. Clin Calcium 22: 67-73.

36. Izumi Y, Aoki A, Yamada Y, Kobayashi H, Iwata T, Akizuki T, et al. (2000) Current and future periodontal tissue engineering. Periodontol 56: $166-87$

37. Yamada Y, Nakamura S, Ito K, Kohgo T, Hibi H, Nagasaka T, et al. (2008) Injectable tissue-engineered bone using autogenous bone marrow-de- rived stromal cells for maxillary sinus augmentation: clinical application report from a 2-6-year follow-up. Tissue Eng Part A 14: 1699-707.

38. Yu H, Yang X, Cheng J, Wang X, Shen SG (2011) Distraction osteogenesis combined with tissue-engineered cartilage in the reconstruction of condylar osteochondral defect. J Oral Maxillofac Surg 69: 558-64.

39. Louis $P$ (2011) Bone Grafting the Mandible. Oral Maxillofacial Surg Clin N Am 23: 209-227.

40. Quereshy FA, Dhaliwal HS, El SA, Horan MP, Dhaliwal SS (2010) Resorbable screw fixation for cortical onlay bone grafting: a pilot study with preliminary results. J Oral Maxillofac Surg 68: 2497-502.

41. Louis PJ, Gutta R, Said-Al-Naief N, Bartolucci AA (2008) Reconstruction of the maxilla and mandible with particulate bone graft and titanium mesh for implant placement. J Oral Maxillofac Surg 66: 235-45.

42. Farzad M, Mohammadi M (2012) Guided bone regeneration: A literature review. J Oral Health Oral Epidemiol 1: 3-18.

43. Chang S, Kuo S, Niu G, Lan C, Cheng W, Yang C (2009) Guided Tissue Regeneration with Use of $\beta$-TCP/Chitosan Composite Membrane. J Appl Polym Sci 112: 3127-3134.

44. Gita V and Chandrasekaran S (2011) Hard and soft tissue augmentation to enhance implant predictability and esthetics: The perio-esthetic approach. J Indian Soc Periodontol 15: 59-63.

45. Le B, Rohrer MD, Prasad HS (2010) Screw "Tent-Pole" Grafting Technique for Reconstruction of Large Vertical Alveolar Ridge Defects Using Human Mineralized Allograft for Implant Site Preparation. J Oral Maxillofac Surg 68: 428-435

46. Zakhary I, El-Mekkawi H, and Elsalanty M (2012) Alveolar ridge augmentation for implant fixation: status review. Oral Surg Oral Med Oral Pathol Oral Radiol 114: S179-S189.

47. Elo J, Herford A, Boyne P (2009) Implant Success in Distracted Bone versus Autogenous Bone-Grafted Sites. J Oral Implantol 35: 181-4.

48. Demarosi F, Leghissa G, Sardella A, Lodi G, Carrassi A (2009) Localised maxillary ridge expansion with simultaneous implant placement: A case series. Br J Oral Maxillofac Surg 47: 535-540.

49. Hwang S, Jung J, Jung J, and Kyung S (2004) Vertical Alveolar Bone Distraction at Molar Region Using Lag Screw Principle. J Oral Maxillofac Surg 62: 787-794.

50. Moghadam HG (2009) Vertical and Horizontal Bone Augmentation with the Intraoral Autogenous J-Graft. Implant Dentistry 18: 230-235. 